\title{
Análisis de Rutas de Transporte de Pasajeros Mediante la Herramienta Network Analyst de Arcgis. Caso Aplicado en la Ciudad de Medellín
}

\author{
Conrado Augusto Serna-Uran \\ Universidad de San Buenaventura \\ conrado.serna@usbmed.edu.co
}

\author{
Jorge Alberto García-Castrillón \\ Especialista en Sistemas de \\ Información Geográfica
}

\author{
Ossman Flórez-Londoño \\ Especialista en Sistemas de \\ Información Geográfica
}

(Tipo de Artículo: Investigación Científica y Tecnologica. Recibido el 24/08/2016. Aprobado el 22/09/2016)

\begin{abstract}
Resumen. El transporte público de pasajeros ocupa un papel importante en las grandes ciudades; teniendo encuenta que la población se encuentra en constante aumento, es relevante analizar este tema y tomar decisiones en función de la optimización de rutas y el mejoramiento del servicio del transporte público. En la actualidad, desde la perspectiva de los viajeros, existen muchas alternativas (modos de transporte) para movilizarse al interior de una ciudad. La elección de qué modo elegir puede estar basada en mucho criterios, tales como: los costos, comodidad, distancia, tiempos de viaje, etc. Alrededor de este tema, muchas ciudades optan por planes de desarrollo que incluyan estrategias de transporte público eficientes, con bajos impactos ambientales y que sea atractivo para los usuarios. En este contexto, el presente artículo pretende abordar una metodología de análisis para evaluar las rutas de transporte público urbano y proponer alternativas que mejoren su desempeño. El análisis es desarrollado para una ruta de transporte público en la ciudad de Medellín utilizando la herramienta de Network Analyst en Arcgis.
\end{abstract}

Palabras claves: Análisis de rutas, transporte público, Network Analyst - ArcGis

\section{Analysis of Passenger Transportation Routes through Network Analyst Tool of Arcgis. Case Applied in Medellin City}

\begin{abstract}
Public passenger transport plays an important role in large cities; given that the population is constantly increasing, it is important to analyze this issue and make decisions based on route optimization and improvement of public transport service. Currently, from the perspective of travelers, there are many alternatives (modes) to move within a city. The choice of how choice can be based on many criteria, such as: cost, convenience, distance, travel time, etc. Around this issue, many cities choose to development plans that include strategies for efficient public transport, low environmental impact and be attractive to users. In this context, this article aims to address an analysis methodology for evaluating urban public transport routes and propose alternatives to improve their performance. The analysis is developed for a public transport route in the city of Medellin using the Network Analyst in ArcGIS.
\end{abstract}

Keywords: Route analysis, public transport, Network Analyst - ArcGis

\section{Introducción}

En muchas ciudades en el mundo, la planificación del transporte público de pasajeros cada vez toma más importancia debido al notable incremento demográfico y económico que se da en muchas regiones. La construcción de infraestructura que de paso a sistemas integrados de transporte parece ser una tendencia en muchas ciudades. Estos sistemas pueden llegar a tener modos de transporte como: buses articulados, trenes, vías peatonales, bicicletas, lo que involucra inversiones de dinero que muchas ciudades pueden no poseer. Por otra parte, la integración del transporte o el desarrollo y fortalecimiento de los modos de manera individual, pueden ser soluciones de corto y mediano plazo que den respuesta a condiciones actuales, pero sean insuficientes comparadas con las tasas de crecimiento en el número de viajes de pasajeros que se presentan en algunas ciudades.

La solución a los problemas de congestión en las ciudades no está en la construcción o ampliación de la malla vial, sino que radica en la planificación y optimización del transporte masivo, que permita satisfacer la demanda de usuarios haciendo un buen uso de los recursos existentes y garantizando la accesibilidad de las personas de diferentes actividades económicas [1].
Además de las inversiones en infraestructura la cual conlleva operaciones de mantenimiento y planificación para mantener unas condiciones óptimas [2] , otra alternativa que puede ser complementaria a esta, es la optimización de los recursos de movilidad con los que cuenta la ciudad. Algunos trabajos de investigación hacen hincapié en este aspecto; por ejemplo Sun, Sun, $\mathrm{Li}, \& \mathrm{Gao}$ [3] hacen referencia a diferentes estudios que se han hecho al respecto, como son: Ceder \& Wilson [4], Baaj \& Wilson [5], Fan \& Machemehl [6], Zhao \& Zeng [7] cuyo objetivo es proponer una optimización simple de los sistemas de transporte masivo. Basados en estos referentes, Sun, Sun, Li, \& Gao elaboran propuestas orientadas a la integración entre el transporte ferroviario y de autobuses usando correlación de variables para definir un corredor vial que optimiza las rutas existentes y define rutas nuevas complementarias al sistema de transporte masivo. Arroyave \& Naranjo [8] proponen un modelo de ruteo para la distribución urbana de mercancías usando plataformas logísticas. Las autoras utilizan un algoritmo Greedy para dar solución al modelo matemático resultante. Otro estudio que también explora heurísticas de solución a problemas de ruteo es el presentado en [9], en el cual se desarrolla un algoritmo hibrido evolutivo para solucionar un problema básico de transporte.

Mauttone, Cancela, \& Urquhart [10] describe el papel importante que juega el transporte público de pasajeros 
en las grandes ciudades y el apoyo en la planificación de los sistemas de transporte público basados en algoritmos y modelos para diseño de rutas y frecuencias de viajes que buscan maximizar el nivel de servicio y minimizar el uso de recursos por medio de restricciones.

\section{Marco Conceptual}

El transporte público de pasajeros debe tener una respuesta que satisfaga las necesidades de los usuarios, para esto es necesario realizar modificaciones en la infraestructura de la ciudad que tiendan a reducir las distancias entre los orígenes y los destinos de cada viaje. Por otro lado, el transporte debe brindar a los usuarios calidad en el servicio, seguridad y tarifas que sean accesibles para todos.

El servicio debe mantener un equilibrio entre el modo de transporte y el usuario, evitando aspectos como el transbordo que puede resultar incómodo para los usuarios y un posible aumento en la congestión e ineficiencia en la oferta por el cruce entre rutas.

Es importante anotar que la infraestructura de la vía es el aspecto más importante y el que menos variabilidad presenta para el funcionamiento de la movilidad urbana, por lo que debe ser optimizado en función de los usuarios de las vías (personas) y no de los vehículos que se desplazan por ellas, evaluando la gestión sobre la demanda, la actuación del servicio de transporte público y el transporte privado, la movilidad de los peatones, el transporte de carga, la infraestructura de las vías, la seguridad del tránsito y la gestión del tránsito.

En este sentido, Ángel Restrepo \& Marín Sepúlveda [11] definen la importancia de las direcciones de las calles y el uso eficaz de la malla vial buscando minimizar eventualidades en las vías que dificulten o imposibiliten el correcto uso para que los costos asociados a los recorridos sean mínimos. En su trabajo buscan obtener el valor de los recorridos mínimos basados en segmentos a transitar para que la ruta sea óptima utilizando algoritmos que determinan la menor distancia entre vértices, como son: el algoritmo de Dijkstra [12], el algoritmo de Bellman-Ford [13], el algoritmos de Johnson de 1977 [14], el algoritmos de Suurballe [15] y el Floyd-Warshall [16] que permiten encontrar los valores de las rutas mínimas de los nodos de un grafo conexo compuesto por arcos dirigidos, no dirigidos 0 mixtos, con pesos positivos, determinando de esta manera los valores de las rutas mínimas absolutas entre la totalidad de los nodos. Luego de la optimización de las rutas, el modelo diseñado por Ángel Restrepo \& Marín Sepúlveda [11], continúan con un sistema de optimización del número de vehículos que permite planificar un número de rutas optimas a partir de las paradas y la demanda en cada una de ellas.

Yang et al. [17] indican la importancia de tener en cuenta la oferta y la demanda del servicio relacionados con la velocidad de los vehículos para realizar un análisis en la red de transporte, la frecuencia de la ruta y los tiempos de viaje considerando la maximización del uso de la ruta y la minimización de los tiempos totales de viajes, que al ser una variable poco predecible se suponen constantes, además de fijar otros supuestos como:

(1) La matriz origen destino de tránsito se fija antes y después de la mejora de las rutas de autobús.

(2) La velocidad del vehículo es fija y la congestión del tráfico en la carretera no es considerada.

(3) El tiempo de espera de los pasajeros es igual a la mitad de la ruta de avance.

(4) El pasajero elige el camino que necesita el número de transferencia mínima de su nodo de origen hasta su nodo destino.

\section{Transporte Público en Medellín}

En el caso de Colombia, el transporte de pasajeros generalmente ha sido manejado por la empresa privada, las autoridades de tránsito evalúan las necesidades de los usuarios y asignan, eficiente o deficientemente, rutas a dichas empresas, las cuales cubren con buses, busetas, microbuses o colectivos.

En el año 1982 se inicia la primera forma moderna de transporte masivo de Colombia, en la ciudad de Medellín, la cual actualmente integra un sistema ferroviario de transporte (Metro), un sistema de cable aéreo que cubre los barrios en las montañas de la ciudad (Metrocable), un sistema de transporte masivo de mediana capacidad (Metroplus) y unas rutas de sistema complementario de minibuses (SIT). En la actualidad se encuentra en construcción una línea tranviaria que favorecerá la zona centro oriental de la ciudad (Tranvía de Medellín). Todo este sistema corresponde a planes urbanísticos del departamento de Antioquia convirtiéndose en un polo turístico y generando desarrollo a su alrededor. En términos de operación, para el cuarto trimestre de 2014 el Sistema Metro de Medellín transportó 86,4 millones de pasajeros, equivalentes al $49,4 \%$ de los usuarios movilizados en el área metropolitana de la ciudad de Medellín, el transporte urbano tradicional contó con 3.383 vehículos en promedio movilizando 88,7 millones de pasajeros, equivalentes al $50,6 \%$ del total de usuarios de la ciudad.

Los usuarios del transporte público se pueden agrupar según el propósito de su viaje [18]. En la última encuesta de origen destino realizada en Medellín se destaca que el $43 \%$ son personas que trabajan y el $24 \%$ son estudiantes para un total del $70 \%$ de los usuarios [19], ver Figura 13. Motivo de viaje, Encuesta Origen Destino (2012). Por otra parte, Alrededor del $28 \%$ de los usuarios utilizan el transporte público colectivo (TPC), además el $55 \%$ de los viajes en transporte masivo presentan por lo menos dos etapas de viaje, caso contrario al TPC donde el $95 \%$ de los viajes se dan en una sola etapa. Otro aspecto importante a tener en cuenta son los tiempos de viaje que para la fecha de la última encuesta de origen destino se promedia en 33 minutos y se concentra en las denominadas horas pico: de 6-8 a.m. y de 5-7 p.m. 


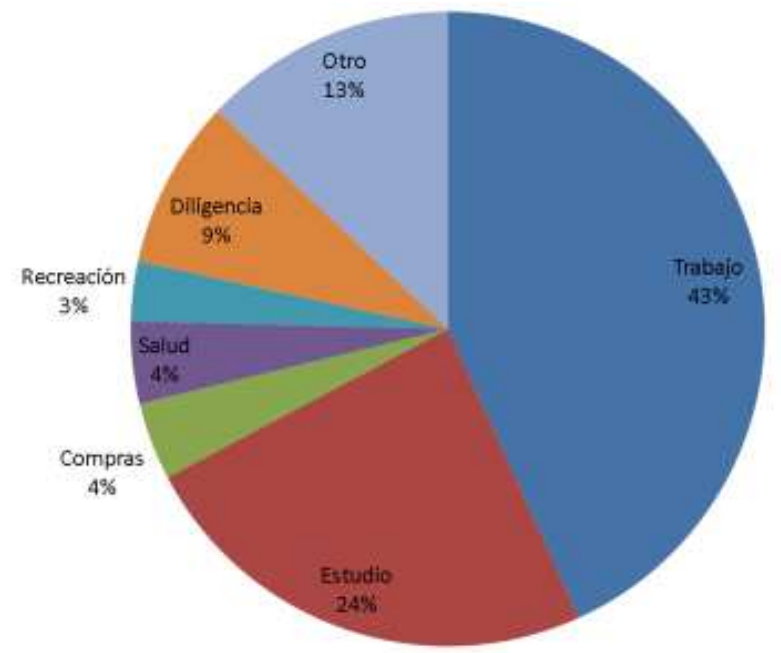

Figura 13. Motivo de viaje, Encuesta Origen Destino (2012)

Los datos que muestra la Alcaldía de Medellín [20] indican que actualmente la ciudad de Medellín cuenta con 40 empresas de transporte público colectivo, 133 rutas de transporte clasificadas en cuatro zonas: NorOriental, Nor-Occidental, Sur-Occidental y SurOccidental, 3703 vehículos de transporte público colectivo matriculado en la SMM y 27 rutas alimentadoras del sistema metro.

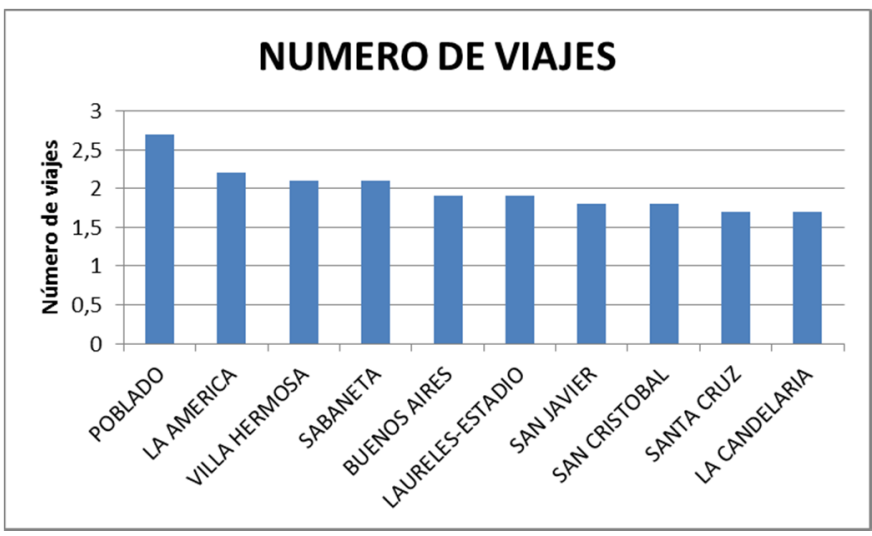

Figura 14. Número de viajes por habitante [19]

Uno de los principales destinos para las personas es el sector de El Poblado con un poco más de 400.000 viajes representado en la Figura 15. Actualmente el sector del Poblado en la ciudad de Medellín cuenta con varias rutas de transporte de pasajeros, las cuales es de gran importancia monitorear constantemente y de acuerdo a las necesidades de las personas, trazar alternativas que aprovechen de mejor forma las condiciones de las vías, el tiempo de recorrido, los costos de viajes entre otros. Es por ello que se hace necesario utilizar herramientas de análisis de rutas que permitan optimizar rutas de transporte en términos de distancia, tiempos, cobertura, etc. A continuación se describe la metodología de análisis empleada en este estudio, con el fin de obtener rutas de transporte que tengan un buen desempeño en estos aspectos

\section{Metodología}

Para minimizar los factores que hacen menos eficientes las rutas de transporte se realiza un análisis ajustado a las necesidades tanto de los usuarios como de las administraciones públicas. El propósito de este análisis consiste en buscar el camino más corto para una ruta de transporte maximizando el número de pasajeros [10]. Este análisis es soportado en el presente estudio a través de un sistema de información geográfica (SIG); como se menciona en [21], estos sistemas se han consolidado en los últimos años como un apoyo importante para la toma de decisiones en áreas como la ingeniería.

La Figura 16 resume de manera general la metodología que será usada en el análisis de una ruta de transporte de pasajeros. Esta metodología está basada en Network Analyst, la cual es una herramienta de análisis de redes proveída por el sistema de información geográfica ArcGis. Inicialmente deben identificarse los diferentes barrios y comunas que conforman la ciudad de Medellín, esto con el fin de hacer un tratamiento apropiado a la Matriz Origen Destino. Posteriormente debe construirse la malla vial de la ciudad con los atributos correspondientes en cada una de las vías (velocidad permitida, restricciones de paso, sentidos, etc.). La información de la malla vial debe ser complementada con los cruces con semáforos presentes en las vías. Una vez se ha hecho la identificación geográfica y de red de la región de análisis se procede con la construcción y evaluación de las rutas.

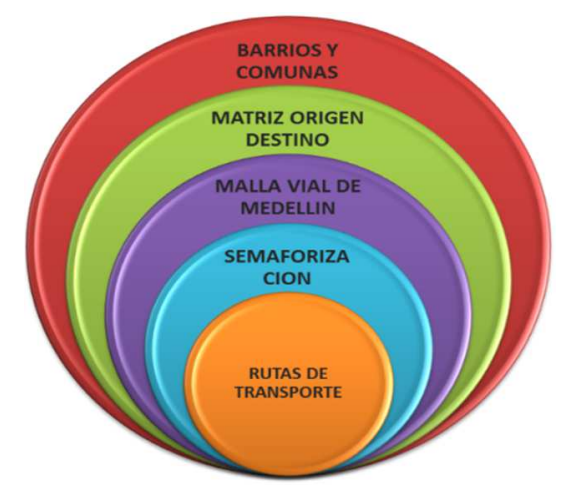

Figura 16. Metodologia para el analisis de rutas

La ciudad de Medellín está dividida en 16 comunas que a su vez se dividen en 249 barrios. Sin embargo, en la Encuesta Origen Destino realizada en Medellín en el 2012, la división de las comunas no se hace por barrio sino por zona SIT que corresponde a la zonificación del Sistema integrado de Transporte (SIT); en total se definen 432 zonas SIT para esta encuesta. Para el análisis aquí desarrollado, se elige una de las comunas que presenta mayores problemas de movilidad, como es la comuna 14, El Poblado, la cual está conformada por 34 zonas SIT. El propósito, por lo tanto, es evaluar una ruta de bus que presta su servicio de transporte en el sector El Poblado, para luego construir una ruta alternativa que mejore la primera en cuanto a condiciones de tiempo y número de personas 
movilizadas, usando para esto información de los viajes que se generan en la zona según la Encuesta Origen Destino realizada en Medellín en el 2012 y la construcción de un dataset de red con las características requeridas para usar las herramientas de optimización de Network Analyst.

Siguiendo la metodología propuesta, se hace la representación geográfica de la malla vial para el sector El Poblado (Shapefile) en el sistema de Coordenadas WGS84 de las vías del sector El Poblado con las respectivas validaciones de topología. La malla vial es complementada con atributos como: sentido de las vías, velocidad máxima permitida, cruces semaforizados, entre otros atributos que puedan afectar el modelo en el momento de generar una ruta óptima. Este proceso y los procesos siguientes, los cuales involucran un manejo del Sistema de Información Geográfico y el análisis de rutas, se resume en la Figura 17.

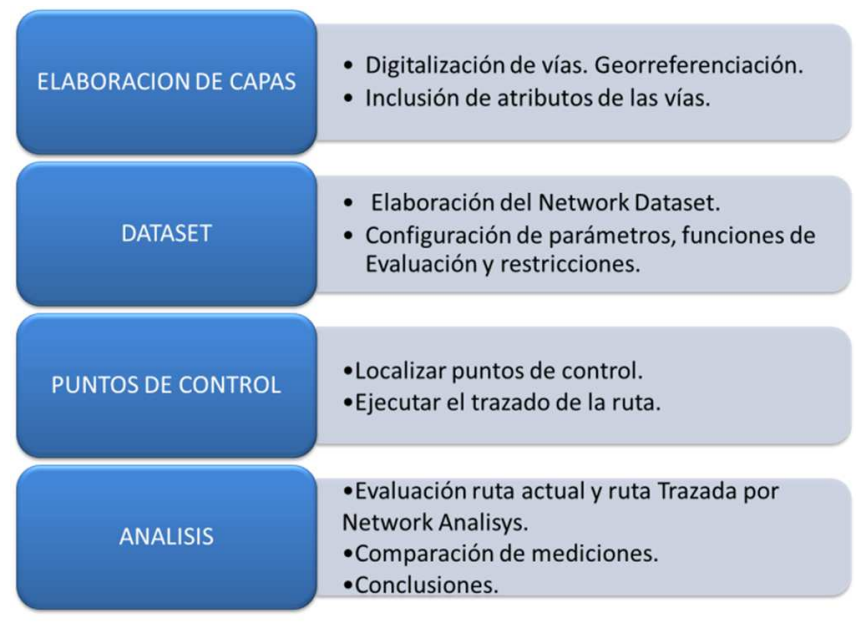

Figura 17. Principales procesos por fase

Para la creación del dataset de red (network dataset) que permita modelar la red de transporte, se ubican nodos en cada una de las intersecciones de las vías. La red de transporte se configura con las funciones de evaluación necesarias para que asimile el sentido de las vías; adicionalmente el Dataset permite la configuración de atributos como velocidad máxima permitida en las vías, semaforización, cruces prohibidos, calles cerradas, entre otros factores que pueden afectar el resultado del modelo. Es importante crear y configurar de una manera adecuada el Network Dataset ya que el algoritmo de Network Analyst se basa en esta red para encontrar una ruta óptima. En la Figura 18 se muestra la malla vial para la zona de estudio.

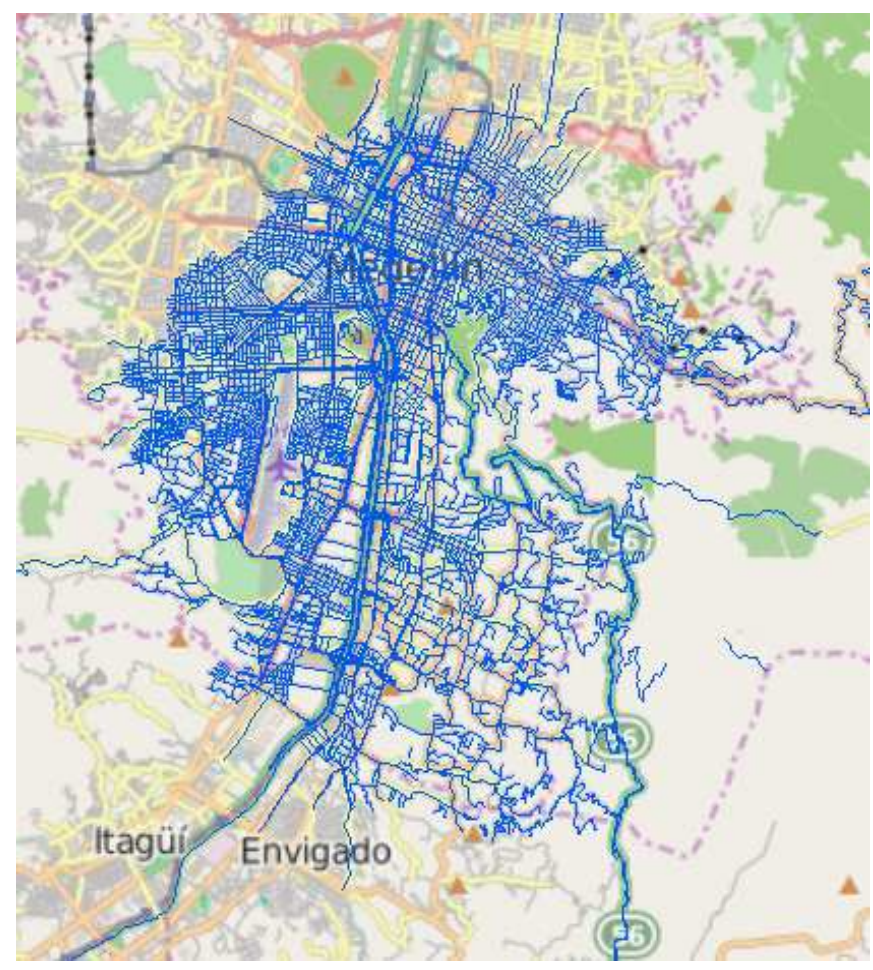

Figura 18. Malla vial El Poblado

La generación de una ruta óptima consiste básicamente en colocar puntos de control basados en una ruta que opere actualmente en El Poblado y ejecutar la herramienta de Network Analyst para que trace una ruta óptima teniendo en cuenta los parámetros y funciones definidas en el Network Dataset. Para el presente análisis se tomó la ruta 133 San Lucas, la cual se enseña en la Figura 19. Esta ruta tiene actualmente una distancia de recorrido de 21.645 metros, en su recorrido total logra cubrir 13 zonas SIT de la encuesta origen destino incluyendo los barrios: Asomadera \#1, Astorga, Barrio Colombia, Bomboná \#1, Castropol, El Poblado, El Tesoro, Los Balsos \#1, Los Naranjos, Manila, San Diego, San Lucas y Villa Carlota, con aproximadamente 4.271 usuarios, en la infraestructura de la vía la ruta pasa por 51 cruces semaforizados.

Por último, el análisis de rutas tiene como objetivo realizar una comparación entre la ruta que opera actualmente y la ruta trazada mediante el algoritmo de optimización que implementa Network Analyst.

En esta fase se realiza una comparación usando la matriz origen destino que indica el número de pasajeros que son transportados de un barrio a otro, tiempos de viaje y otros factores que indiquen una mejoría a la ruta actual. Finalmente se muestran los resultados del análisis comparativo entre las rutas y los aspectos que mejoraron usando Network Analyst. 
Ingenierias USBMed, Volumen 7, No. 2, Julio-Diciembre 2016

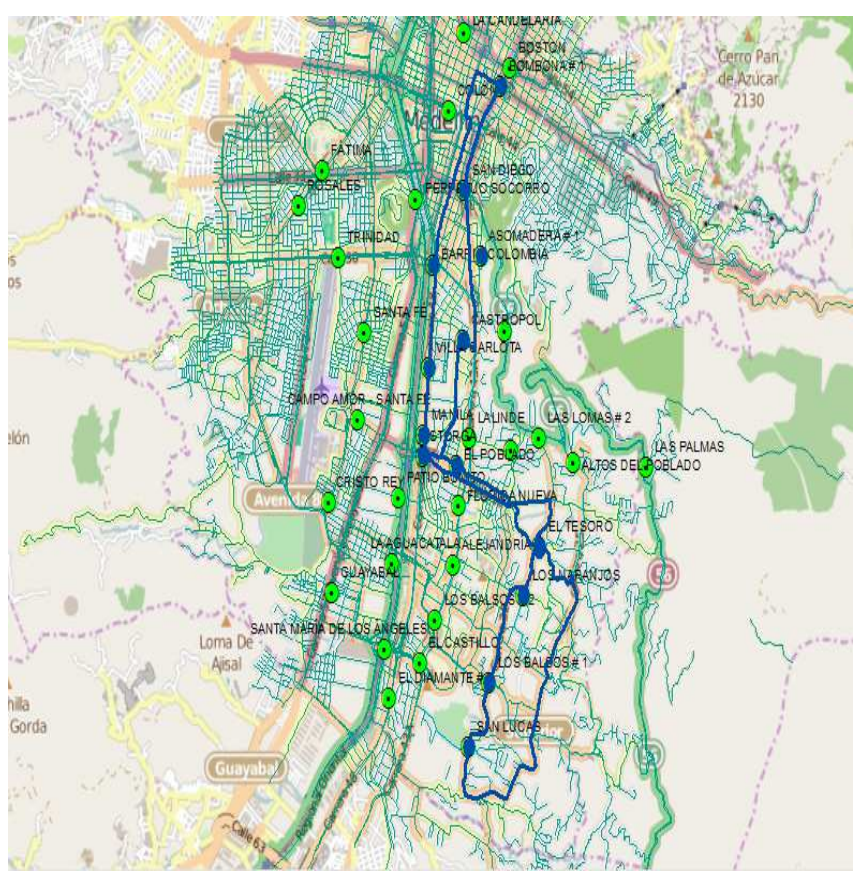

Figura 19. Ruta seleccionada 133 El Poblado

\section{Análisis de Ruta.}

Los atributos de la red proporcionan elementos de red básicos como la distancia de cada tramo de vía, la direccionalidad de las calles (un sentido o doble sentido) y el nivel de jerarquía (autopista, arterial, carretera local). Para tomar la mejor ruta se toma como impedancia la distancia.

Se identifican los nodos principales para el análisis de las rutas comenzando por el nodo uno (1) o nodo de inicio, un nodo de finalización y varios nodos a escanear por donde se indican las paradas mínimas o nodos alcanzados por la ruta. En total se definen 14 nodos obligando a que el modelo de ruta cruce por las zonas de origen destino como puntos de control obligatorios y que inicialmente son cubiertas por la ruta inicial, ver Figura 20.

Una vez seleccionados los nodos se da una solución, generando una nueva ruta con una distancia de recorrido de 17.930 metros, que logra cubrir 18 zonas SIT: Alejandría, Asomadera \#1, Astorga, Barrio Colombia, Bomboná \#1, Boston, Castropol, El Castillo, El Poblado, El Tesoro, Florida Nueva, La Linde, Los Balsos \#1 y \#2, Los Naranjos, Manila, San Diego y San Lucas, de aproximadamente 7.167 usuarios. Esta nueva ruta pasaría por 52 cruces semaforizados. La Figura 21 muestra la ruta obtenida.

Se realiza una comparación con los resultados de las dos rutas (ruta original vs. ruta optimizada) calculando los tiempos de viaje para cada tramo con una velocidad promedio entre los $17 \mathrm{~km} / \mathrm{h}$ y $22,1 \mathrm{~km} / \mathrm{h}$. Se puede evidenciar un ahorro en cuanto a distancia y tiempo de recorrido de aproximadamente un 17\%. El número de cruces semaforizados no varía considerablemente pasando de 51 a 52 cruces. La Figura 22 hace una comparación entre la ruta actual (línea delgada negra) y la propuesta (línea gruesa purpura).

La Tabla 12 resume los resultados de la optimización realizada y la economía de la ruta inicial comparada con la ruta generada con el método de solución y que permite obtener un ahorro de cerca del $21 \%$ en la distancia recorrida al igual que en los tiempos de desplazamiento considerando condiciones especiales de la vía, como velocidades constantes, flujo de la movilidad continua. Además se muestra un incremento en los puntos de origen destino (zonas SIT) cerca del $28 \%$ lo que permite maximizar el número de usuarios aproximadamente en un $40 \%$ que posiblemente harían uso de dicha ruta.

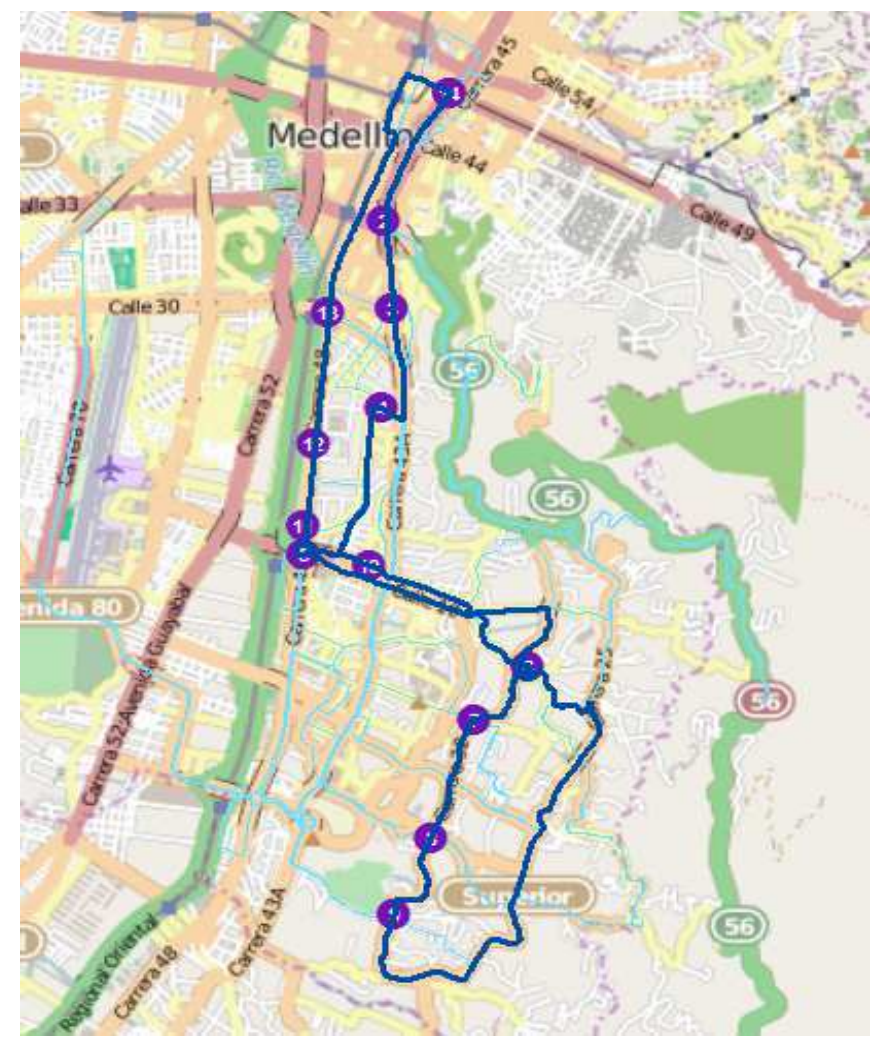

Figura 20. Nodos seleccionados 


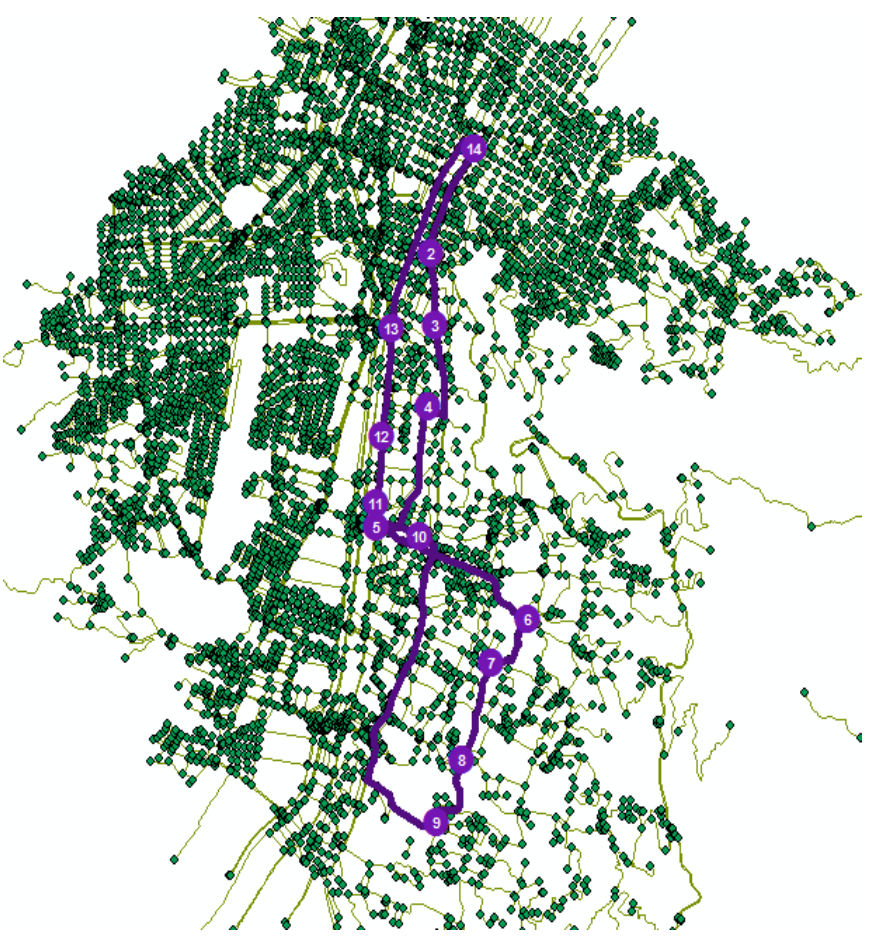

Figura 21. Ruta optima vs ruta inicial

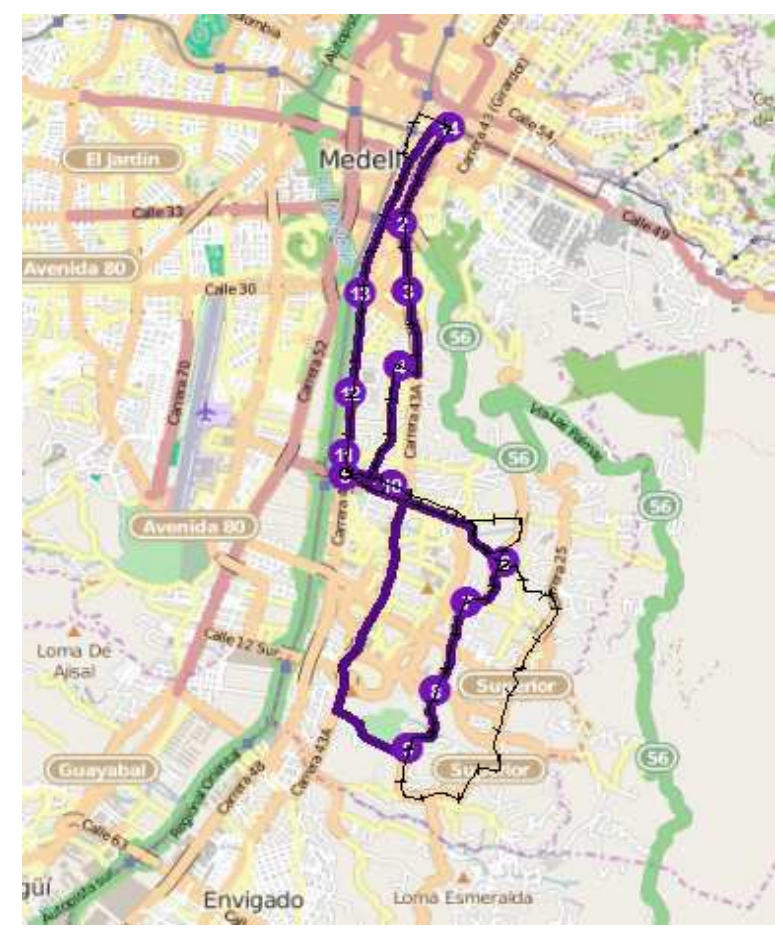

Figura 22. Análisis de direcciones

Tabla 11 Datos de análisis

\begin{tabular}{|c|c|c|c|c|c|c|}
\hline & Distancia $(\mathrm{km})$ & Cruces semaforizados & $\begin{array}{l}\text { Tiempo de } \\
\text { recorrido (min) }\end{array}$ & $\begin{array}{l}\text { Velocidad } \\
\text { promedio }(\mathrm{km} / \mathrm{h})\end{array}$ & $\begin{array}{l}\text { Zonas origen } \\
\text { destino }\end{array}$ & $\begin{array}{l}\text { Usuarios de } \\
\text { rutas }\end{array}$ \\
\hline Ruta original & 21.645 & 51 & 673935 & 20 & 13 & 4271 \\
\hline Ruta optima & 17.93 & 52 & 53.79 & 20 & 18 & 7167 \\
\hline Diferencia & $-21 \%$ & $2 \%$ & $-21 \%$ & $0 \%$ & $28 \%$ & $40 \%$ \\
\hline
\end{tabular}

\section{Conclusiones}

En el desarrollo del presente trabajo y gracias a los resultados obtenidos, fue posible verificar el potencial que poseen los sistemas de información geográfica para realizar análisis de redes de transporte en escenarios urbanos. Su uso, además, puede facilitar la integración de análisis espaciales y geoestadísticos que han sido ignorados en muchos modelos logísticos y con los cuales se mejoraría la toma de decisiones en este campo.

El método propuesto a partir del uso de Network Analyst permitió integrar múltiples objetivos en el modelo de solución como el número de usuarios potenciales en la ruta, velocidad, tiempos de recorrido, distancias, lo que demuestra. Esta integración facilitó la comparación de múltiplos criterios para evaluar la eficiencia de la ruta obtenida en relación a la ruta de transporte que se realiza actualmente

Aun cuando se tomaron restricciones y condiciones del modelo real para determinar una ruta optima mediante Network Analyst, como los cruces, la velocidad promedio de la vía, etc., es necesario tener en cuenta otros factores que intervienen en los procesos de transporte como altimetría, condiciones de la vía, trafico, etc., con el fin de lograr soluciones más robustas y adecuadas a las condiciones reales.

\section{Referencias}

[1] P. Jaramillo Álvarez, C. A. González Calderón y G. González calderón, «Route optimization of urban public transportation» Dyna, vol. 180, no 80, pp. 41-49, 2013.

[2] J. A. Zapata y G. J. Cardona, «Aplicación de los sistemas de información geográfica para la gestión de la malla vial de la ciudad de Medellín» Ingeniería USBMed, vol. 3, № 2, pp. 70-84, 2012.

[3] Y. Sun, X. Sun, B. Li y D. Gao, «Joint optimization of a rail transit route and bus routes,» Procedia - Social and Behavioral, vol. 96, no 96, p. $1218-1226,2013$.

[4] A. Ceder y N. Wilson, «Bus Network Design» Transportation Research Part B, p. 331-344, 1986.

[5] M. Baaj y N. Wilson, «An Al-based approach for transit route system planning and design" Journal of advanced transportation, p. 187-210, 1991.

[6] W. Fan y R. B. Machemehl, «Optimal Transit Route Network Design Problem with Variable Transit Demand: Genetic Algorithm» Journal of Transportation Engineering, p. 40-51, 2006.

[7] F. Zhao y X. Zeng, «Optimization of transit route network, vehicle headways and timetables for large-scale transit networks" European Journal of Operational Research, p. 841-855, 2008.

[8] M. R. Arroyave y V. V. Naranjo, «Desarrollo de un modelo de distribución urbana de mercancías con plataformas logísticas aplicado a la ciudad de Medellín» Ingeniería Usbmed, vol. 5, no 1, pp. 67-76, 2014. 
[9] M. D. Arango y C. A. Serna, «A memetic algorithm for the traveling salesman problem» IEEE Latin America Transactions, vol. 13, no 8, pp. 2674-2679, 2015

[10] A. Mauttone, H. Cancela y M. U. Urquhart, «Diseño y optimización de rutas y frecuencias en el transporte colectivo urbano, modelos y algoritmos» Universidad de la República Facultad de Ingeniería, Uruguay, 2010.

[11] P. L. Ángel Restrepo y L. F. Marín Sepúlveda, «Un método computacional para la obtención de rutas óptimas en sistemas viales,» DYNA, vol. 78, oㅜ 167, pp. 112-121, 2011.

[12] K. Murota y A. Shioura, «Dijkstra's algorithm and L-concave function» Math. Program, p. 163-177, 2014.

[13] K. R. Hutson, T. L. Schlosser y D. R. Shier, «On the Distributed Bellman-Ford Algorithm and the Looping Problem» Informs journal on computing, pp. 542-551, 2007.

[14] C. J. Ong y E. G. Gilbert, «Fast Versions of the Gilbert-JohnsonKeerthi Distance Algorithm: Additional Results and Comparisons» Transactions on Robotics \& Automation, pp. 531-540, 2001.

[15] T. Gomes, C. Simoes y L. Fernandes, «Resilient routing in optical networks using SRLG-disjoint path pairs of min-sum cost» Telecommunication Systems, pp. 737-749, 2013.

[16] P. Höfner y B. Möller, «Dijkstra, Floyd and Warshall meet Kleene» Formal Aspects of Computing, pp. 459-476, 2012.

[17] S. Yang, X. Suna, B. Lia y D. Gaob, «Joint optimization of a rail transit route and bus routes in a transit corridor,» Procedia - Social and Behavioral Sciences, vol. 96, p. 1218 - 1226, 2013.

[18] D. Heinrichs y J. S. Bernet, «Public Transport and Accessibility in Informal Settlements: Aerial Cable Cars in Medellín, Colombia» Transportation research procedia, vol. 4, pp. 55 - 67, 2014.

[19] Área Metropolitana Valle de Aburrá, «Encuesta origen destino de hogares 2012» Medellín, 2012.
[20] Secretariá de Movilidad de Medellín, «Alcaldia de Medellín» 2013. [En línea]. Available: https://www.medellin.gov.co/movilidad/cifras-estudios. [Último acceso: 0305 2015].

[21] C. E. Durango y C. M. Zapata, «Una representación basada en Semat y RUP para el Método de Desarrollo SIG del Instituto Geográfico Agustín Codazzi» Ingeniería USBMed, vol. 6, no1, pp. 24-37, 2015.

[22] DANE, Transporte urbano de pasajeros, Bogotá D.C., 2015.

[23] Secretaría de Tránsito y Transporte Alcaldía Mayor De Tunja, "Herramienta de transporte terrestre urbano de pasajeros y carga. Simulación del efecto de proyectos de infraestructura y políticas de transporte» Tunja, 2012.

[24] H. C. Antonio Mauttone, «Diseño y optimización de rutas y frecuencias en el transporte colectivo urbano, modelos y algoritmos» Uruguay.

[25] Unidad de servicios de Infraestructura, «Políticas integradas y sostenibles de movilidad: revisión y propuesta de un marco conceptual» Boletín FAL, vol. 7, no 7, p. 9, 2013.

[26] A. Tobón y D. Galvis, «Análisis sobre la evolución reciente del sector de transporte en Colombia," Perfil de coyuntura económica, № 13, pp. 147 - 163, 2009.

[27] J. Seguí, M. Ruiz, F. Escalas, A. Bauçà, F. Guaita, J. Pacheco y C. Delgado, «La planificación de rutas de trasporte escolar a través de un sig: el proyecto sigtebal» Universidad de Burgos, Mallorca.

[28] J. J. Posada Henao, V. Farbiarz Castro y C. A. González Calderón, "Análisis del "pico y placa" como restricción a la circulación vehicular en Medellín basado volúmenes vehiculares,» DYNA, vol. 78, no 165, pp. 112 - 121, 2011.

[29] F. Fuquene, «Estudio de movilización de pasajeros en rutas urbanas de la ciudad, mediante aforos de registradoras» Medellin, 2013. 